This item was submitted to Loughborough's Research Repository by the author.

Items in Figshare are protected by copyright, with all rights reserved, unless otherwise indicated.

\title{
A meta-analysis of biological variation in blood-based therapy as a precursor to bio-manufacturing
}

\section{PLEASE CITE THE PUBLISHED VERSION}

http://dx.doi.org/10.1016/j.jcyt.2016.01.011

\section{PUBLISHER}

Elsevier (@ International Society for Cellular Therapy)

\section{VERSION}

AM (Accepted Manuscript)

\section{PUBLISHER STATEMENT}

This work is made available according to the conditions of the Creative Commons Attribution-NonCommercialNoDerivatives 4.0 International (CC BY-NC-ND 4.0) licence. Full details of this licence are available at: https://creativecommons.org/licenses/by-nc-nd/4.0/

\section{LICENCE}

CC BY-NC-ND 4.0

\section{REPOSITORY RECORD}

Thurman-Newell, Jamie, Jon Petzing, and David Williams. 2019. "A Meta-analysis of Biological Variation in Blood-based Therapy as a Precursor to Bio-manufacturing”. figshare. https://hdl.handle.net/2134/21083. 
A Meta-analysis of Biological Variation in Blood-based Therapy as a Precursor to Bio-Manufacturing.

6

7

8

Jamie. A. Thurman-Newell, Jon N. Petzing, David J. Williams

9

Healthcare Engineering Group, Centre for Biological Engineering, Holywell Park, Loughborough University, LE11 3TU, UK.

17 Funding for this work was provided by a UK EPSRC Grant ( EP/FS00491/1 ) as part of the 18 Doctoral Training Centre for Regenerative Medicine

19 


\section{Abstract}

22 Currently cellular therapies, such as haematopoietic stem cell transplantation ( HSCT ), are

23 produced at a small scale on case-by-case basis, usually in a clinical or near-clinical setting.

24 Meeting the demand for future cellular therapies will require a robust and scalable

25 manufacturing process that is either designed around, or controls the variation associated with

26 biological starting materials.

27 Understanding variation requires both a measure of the allowable variation ( that does not negatively affect patient outcome ) and the achievable variation (with current technology ). The prevalence of HSCT makes it an ideal case study to prepare for more complex biological manufacturing with more challenging regulatory classifications.

31 A systematic meta-analysis of the medical literature surrounding HSCT has been completed of which the key outcomes are;

- The range of transplanted CD34+ cells / $\mathrm{kg}$ can be up to 6 orders of magnitude around the median for allogeneic procedures and 4 orders of magnitude for autologous procedures

- No improvement in variation encountered over a period of thirty years

- As study size increases the amount of variation encountered increases

38 A more detailed, stratified source from a controlled single site clinical centre is required to

39 further define a control strategy for the manufacture of biologics. 


\subsection{Introduction}

41 Cellular therapies are currently produced in small batches, typically in a clinical setting, under special legislation such as the European Hospital Exemption Clause and the FDA's Investigational New Drug Exemption. These small batch sizes will struggle to meet future demand, so an appropriate bio-manufacturing process is likely to be required to replace some or all of the currently predominately manual processing. A quality by design approach to process control that is based around, or controls, the variation inherent to biological starting materials, is anticipated as being a key prerequisite to bio-manufacturing at scale and an ongoing challenging prospect for entrepreneurs, manufacturers and regulators alike.

Understanding biological variation requires both a measure of the;

- Allowable variation, that does not impinge on safety or efficacy

- Achievable variation, as a result of our current level of technology and skill

The allowable variation is based upon the specification, set by the prescriber, which details the limits of variation that a product must remain within to avoid negatively affecting patient outcome. The achievable variation is based upon the tolerance of the process / machine, set by engineers, which is based upon the ability of the process to cope with this variation. Furthermore, an understanding of the variation in the starting material, as a result of the patient/donor population, the isolation techniques and the previous conditioning regimes, is required. Together, this knowledge will ultimately inform strategies to account for this variation, including how to address the issue of comparability - demonstrating that the process remains the same after a change, which can include distributed manufacture at multiple locations, whilst still remaining cost-effective ${ }^{1}$ and within tolerance. known efficacy, at scale. This has the potential to increase the quality of the product

64 ( therefore maximising patient longevity and quality of life ), or maintain the quality of the product but at a reduced cost ${ }^{2}$. 


\subsection{Exemplar}

68 Haematopoetic stem cell therapy ( HSCT) is one of the few cellular therapies currently in

69 routine use $\mathrm{e}^{3}$, with a proven historical track record, and is relatively well established

70 worldwide. The therapeutic potential of this therapy originates from the haematoepoetic

71 progenitor cells - a self-renewing precursor cell that has the potential to become a number of specialised cell and tissues. HSCT utilises the unique properties of these cells, isolated from peripheral blood, bone marrow or cord blood for clinical applications such as the revivification of a patient's bone marrow following potent chemotherapy or radiotherapy.

The prevalence of HSCT is one of the reasons it was chosen as an exemplar to benchmark the variation encountered in cellular therapies. It is a secondary therapeutic ( the chemotherapy or radiotherapy is the primary therapeutic ) but it occupies a unique regulatory niche in that it is 'minimally manipulated', so has the potential as a case study to inform process design for more complex bio-manufacturing of products that are more than minimally manipulated, and fall into more challenging regulatory classifications such as Advanced Therapy Medicinal Products ( ATMPs ) ${ }^{4}$.

\subsection{Variation Meta-Analysis Methodology}

The objective of this research was to determine the baseline extent of variation encountered for HSCT, under the practise of medicine, because this therapy is manufactured and applied within a clinical setting. This research expands upon previous work ${ }^{5}$, by adding greater resolution, and examining the challenge from a nascent biomanufacturing perspective.

This analysis was designed to; examine the medical literature on HSCT for collected / transplanted cell metrics ( such as total nucleated cell count or CD34+ cell count - a cell surface marker present on haematopoietic stem cells ), examine and report the extent of variability in these metrics within and between these studies, and if sufficient detail was present, correlate this variation with donor / patient metrics, processing methodology and clinical centre, amongst other variables.

94 This work is intended to compliment a clinical case study by providing a platform for discussion between stakeholders, and a global picture to compare with single centre study 
data thus providing a demonstration of the current 'state of control'. The research presented here focuses on the results of the literature meta-analysis.

Online databases and health resources ( Pubmed and Web of Knowledge ) were used to search the literature for a number of pre-determined keywords, medical search headings

100 ( MeSH ) and publication dates. Articles were restricted to English language, unless a native translation was provided, and only refereed journals were used ( conference proceedings, for example, were excluded ).

The abstracts of the resultant studies were then screened for the likelihood of containing applicable data, such as clinical trials or outcomes studies. Eligible publications were then obtained in full and stored locally, and given a unique identifier that could be linked to the database for future reference. These publications were then manually examined for patient, donor and graft measurements. A pre-determined checklist of measurements had been previously created for this task, from mind-mapping and stakeholder discussion ( see Table 1 ). A number of primary characteristics, such as the presence of TNC count or CD34+ cell count, were mandatory for studies to pass through to the data collection stage. At this stage,

111 data was transferred and stored next to the identification number within a database. MS

112 Excel and IBM SPSS 22.0 ( New York ) were used for the analysis.

\section{$113 \quad 4.0$ Results}

1145,458 peer-reviewed journal articles ( previously 3,190 ${ }^{5}$ ), between 1980 and 2015, were 115 identified. This resulted in 269 ( previously $126^{5}$ ) articles that contained 491 observations 116 included donor, methodological and graft variables.

117 Variation was measured from reported cell metrics, of which total nucleated cell count 118 ( TNC) and CD34+ cell count were most prevalent. These were reported as TNC / kg and 119 CD34+ cells / kg patient bodyweight. Interquartile ranges were often provided in lieu of 120 range data within articles, but were not recorded as they are not representative of the true value of biological disparity and avoid up to $50 \%$ of possible variation. Cord blood and paediatric datapoints were removed ( due to low incidence level) so this dataset represents

123 adult bone marrow and peripheral blood derived material only. It should be noted that in certain figures the $x$ axis title refers to the unique identifying number given to each valid 
125 study, however data in each of these figures is discontinuous and consequently explicit

126 numbering has been omitted.

127 Biological metrics, such as CD34+ cell count, were heavily negatively skewed, probably due

128 to the presence of these cells being host-state dependant or the minimum collection criteria.

129 Non-parametrically distributed data requires a different statistical approach - for example

130 Spearmans Rank Order Correlation ( SROC) was used for bivariate analysis, rather than the

131 traditional Pearson Product-Moment Correlation used in normally distributed data. The

132 value for SROC refers to the strength and direction of the correlation, the $p$-value refers to

133 the statistical significance of the test, and the $n$-value refers to the number of data-points

134 included in the analysis.

135 The results of the meta-analysis are presented as a summary in italics which prefixes the 136 explanation that follows.

\subsection{Overall Variation}

Under the practise of medicine, the variation currently encountered in HSCT ( represented in this sub-population ) can be between one and four orders of magnitude of the median reported amount.

The range of both collected CD34+ cells ( cCD34) and transplanted CD34+ cells ( tCD34) was calculated for each study sample. An open-high-low-close (OHLC) chart ( Figure 1 ) was used to plot each representative samples' median, minimum and maximum cCD34, and tCD34. This format was used for biological data because it is a visually succinct method of demonstrating the extent of variation with respect to the median, and with other studies. This figure represents the overall variation for (a) cCD34 and (b) $\operatorname{tCD} 34$ ( $n=106$ and $n=303$, respectively ). Each central point represents the median value reported within a particular study, and the vertical lines are indicative of the ranges reported. Point $1 \mathrm{~b}(\mathrm{i})$ on Figure 1 exhibits no obvious characteristics that would differentiate itself from other studies ( as paediatric donors or cord-blood sourced transplants have already been excluded ).

A number of studies have reported a minimum of zero collected or transplanted cell counts which are very particular extremes. It is unlikely this is a true value of sero, 
but rather that insufficient cells were mobilised and was deemed unviable in the study. These values, as much as they must be considered as a representation of an individuals' personalised medicine, skew the overall analysis of variation considerably. Including the zero values the variation is up to 9 orders of magnitude, whilst excluding them the variation is up to 6 orders of magnitude. For this discussion only quantifiable collected / transplanted cell counts have been used -

\subsection{Stratification of the Overall Variation into Collected and Transplanted}

Allogeneic-derived material can vary up to 6 orders of magnitude of the median cell count, whilst autologous-derived material can vary up to 4 orders of magnitude.

Figures 2 and 3 stratify CCD34 / kg / study and tCD34 / kg / study into transplant type

The variation in $\mathrm{tCD} 34$ is;

- up to six orders of magnitude of the median value for allogeneic therapy ( $1.00 \mathrm{e} 3$ cells / $\mathrm{kg}$ to $1.21 \mathrm{e} 9$ cells $/ \mathrm{kg}, n=188$ )

- up to four orders of magnitude of the median value for autologous therapy ( $6.00 \mathrm{e} 4$ cells $/ \mathrm{kg}$ to $3.00 \mathrm{e} 8$ cells $/ \mathrm{kg}, n=110$ )

As a result, autologous therapy appears considerably more manageable from a variation perspective than allogeneic therapy. Further stratification will be required to determine the reason for the greater amount of variation present within allogeneically sourced material. 

community's ability to control variation over a thirty year time period. in which the study commenced. This is the stage where the protocols are established and therefore represent the technology / methodology typical of the time. The relative lack of data points between 2010 and 2015 has been attributed to studies in progress, and not yet reported. The correlation between range of CD34+ cells / kg / study and study year was examined using SROC, for both collected and transplanted cells, to alleviate the Cluster Illusion from data interpretation, between 1980 and 2015. The application of SROC was confirmed by visual inspection and a ShapiroWilks normality test $(p>0.05)$. Preliminary analysis showed the relationship to be monotonic.

There was a weak statistically significant correlation between range of cells, and time for both collected $(S R O C=-0.295, p<0.003)$ and transplanted cells $(S R O C=-0.178$, $p<0.003$ ). There are a number of other factors that may contribute towards a correlation rather than just starting year ( such as incremental technology advances, or institutional experience), however improvement was expected. Additionally the number of unknowns ( of which some are summarised in Section 5: Meta-Analysis Limitations ) are enough that any correlation can be argued to be insignificant or circumstantial.

\subsection{Variation by Country of Study}

There is insufficient resolution to discern any specific advantage in terms of variation between any clinical centre or country.

This meta-analysis contains observations from 32 different countries and by multinationals, which are represented by 143 clinical centres. A Pareto Analysis was previously carried out on the meta-analysis database to discern the leading contributors within this dataset. The Pareto Rule - often called the 80/20 Rule refers to the observation that $80 \%$ of the effect is due to $20 \%$ of the factors. This identified ten countries that had significant presence and were subsequently used to 
plot another OHLC chart ( Figure 5 ). Only tCD34 has sufficient data-points within the dataset to plot with significance.

For each country, the number of centres contributing towards the result has been annotated. It is difficult discern differences between countries without much greater resolution, because each of these centres will probably operate under different practises / equipment / staff, and target different indications and patients. However, there is sufficient evidence that biological variation is a global concern.

\subsection{Variation as a Function of Study Size}

As the number of donor / patients involved in a study increased, the amount of variation also increased.

Figure 6 is a stratified scatter diagram of the range of tCD34 / kg / study against the number of patients / donors in the study. SROCs were carried out for each subsection and have been included in this diagram ( $a-f)$. These statistically significant correlations demonstrate that as the size of the study increases ( and therefore the number of donors and products involved ) the total variation per study increases. From a variation perspective this not only indicates that the current labbased production methods find it increasingly difficult to process large volumes of product in a consistent manner, but will most probably be unsuitable for large scale production of a future therapeutic.

\subsection{Meta-Analysis Limitations}

Due to the disparate and aggregated nature of how public data is reported and subsequently collected in this study, a number of limitations must be considered when regarding these results.

- Outliers. In biological manufacturing, 'outliers' can be representative of the heterogeneity of an individual's biological state ( and/or their personalised medicine ), so cannot be disregarded.

- Sampling Variation. There may be an element of author bias which may have resulted in data-containing studies being overlooked, but given the overall 
spread of the data the authors do not anticipate any significant shift in statistical behaviour.

- Duplicate Data. Due to conglomerate groups, such as the European Bone Marrow Transplant Group ( EBMT ), there is a risk of overlapping data. This is a problem that using dedicated raw data will not encounter.

- Patient versus Donor Weight. cCD34 and tCD34 were reported as cells per kg of patient bodyweight, ideally they would have to be first normalised to donor bodyweight - ( in autologous therapy, this is not an issue because the patient is also the donor ) -for allogeneic therapy this would be an instance of adding distance from the true value.

- Paediatric Analysis. This meta-analysis contained a total of 18 paediatric donor based studies and therefore a separate analysis regarding children has not been made, or a comparison between adult and paediatric cCD34 and tCD34. However importantly, autoimmune disease, inherited metabolic disorders and gene therapy are all prime targets for paediatric therapy ${ }^{6}$ and an understanding of paediatric variation would be beneficial.

- Mobilisation / Conditioning Regime. These are difficult to quantify because they vary on a case-by-case basis, in terms of dosage, regime, drugs, clinician ${ }^{7}$ and a combination of these. Additionally, details were not uniformly reported. These regimes are crucial factors ${ }^{8}$ in the content and quality of the starting material for HSCT and proper characterisation and stratification is vital to a better understanding.

- Apheresis / Aspiration Processes. Details regarding the instrumentation used for apheresis, flow rates, number of procedures or extractions were rarely reported. Furthermore it is not clear from the literature whether $\mathrm{CCD} 34$ is taken from the total collected cell count, or from a particular stage - or how many extractions were used. It is common clinical practise to pool individual products but it is unclear which ( if any ) of the reported metrics are pooled products. The number of collections is also a factor in addition to patient health ${ }^{9}$. 
- Cryopreservation and Transport. It is accepted that cryopreservation causes losses in TNC, viability and CD34+ cell numbers ${ }^{10}$ but it is unclear as to whether this loss, is a contributing factor towards variation.

- Multiple Centres. There are a number of centres with multiple observations within the dataset, but not sufficient numbers to make meaningful statistical observations regarding the variation between and within centres. The use of raw data from clinical centres would be needed to allow this comparison.

- Raw Materials versus Starting Materials. Another variable are additional chemicals added to the product. The UK MHRA has defined these as 'raw materials', whilst the biological component is the 'starting material' and it is important to understand the effect both have on each other and the process.

- Indication. Patient disease state was too generalised to stratify from the dataset. 


\subsection{Conclusion and Summary}

281 This meta-analysis of publically available data has been developed to provide a broad-scope demonstration of the challenge that biological input variation imposes on the potential for controlling large scale bio-manufacture and will impose when manufacturing Advanced Therapy Medicinal Products. The primary output from this analysis are results that represent the extent of biological variation in both collected and transplanted material for a clinical sub-population.

This meta-analysis has determined that;

- The current variation encountered under the practice of medicine for haematopoietic stem cell therapy can be up to six orders of magnitude of the median dose. This level of variation would be unmanageable from a biomanufacturing perspective.

- Comparing the ranges of collected and transplanted CD34+ cells over time, there is It is clear that to make the next decisions on an appropriate bio-manufacturing strategy, a stratifiable data source with greater resolution will be required; such as patient databases and centre-specific clinical records. Without this raw data, quantification of sources and extent of variation contributed by each variable will remain informed speculation.

303 Consequently the next step in this research is the acquisition and analysis of high quality datasets from clinical petitioned national health sources, where the data represents individual cases, not summaries. This will allow stratification of the contributions of variables such as donor age or weight, process parameters and indication and an increased rigour and quality in the reported results. This will also allow access to absolute numbers of cells per collection / product. Additionally, using datasets from specific centres will allow 
309 normalisation of the variation with respect to centre specific factors, such as geographical,

310 surgical, clinical and operator variation, because these should remain constant within a

311 single centre.

312 Variation cannot be eliminated completely, but it will need to be brought to a state where

313 the differences in the product do not negatively affect patient outcome, and is a key step in

314 establishing product specifications and achievable manufacturing tolerance ranges. To meet

315 this challenge, cross-disciplinary collaboration between medical and engineering fields will

316 be crucial $^{11}$; sharing their combined experience will enable strategies required to

317 accommodate and control variation for production to accommodate large numbers of

318 patients - either in large volume in concentrated facilities, or smaller volumes at multi-site

319 centres $^{1}$. 
7.0 Acknowledgements

321

Competing interests: the authors have no competing interests. Funding for this work was provided by a UK EPSRC Grant ( EP/FS00491/1) as part of the Doctoral Training Paul Hourd for their input. 
327 Table 1: Meta-Analysis Search and Retrieval Methodology

328 Figure $1 \quad$ Range of CD34+ Cell Count / kg / Study for;

a) Collected Cells

b) Transplanted Cells

Figure 2

Range of CD34+ Cell Count / kg / Study for;

Autologous Peripheral-sourced transplants

Figure 3 Variation in Transplanted CD34+ Cell Counts / kg / Study stratified into;

334

335

336

337

338

339

340

341

342

343

344 a) Autologous peripheral

b) Autologous bone marrow

c) Autologous mixed source

d) Allogeneic peripheral

e) Allogeneic bone marrow

f) Allogeneic mixed source

Figure $4 \quad$ Range of CD34+ Cells / kg / Study Against Time stratified into;

a) Collected Cells

b) Transplanted Cells

Figure $5 \quad$ Range of transplanted CD34+ cells / kg / Study between Countries

Figure 6 Range of transplanted CD34+ cells / kg / Study against Study Size stratified into;

a) Autologous peripheral

b) Autologous bone marrow

c) Autologous mixed source

d) Allogeneic peripheral

e) Allogeneic bone marrow

f) Allogeneic mixed source 
1. Hourd P, Ginty P, Chandra A, Williams DJ. Manufacturing models permitting roll out/scale out of clinically led autologous cell therapies: Regulatory and scientific challenges for comparability. Cytotherapy. 2014;16(8):1033-1047. doi:10.1016/j.jcyt.2014.03.005.

2. Escalante EJ. Quality and Productivity Improvement: A Study of Variation and Defects in Manufacturing. Qual Eng. 1999;11(3):427-442. doi:10.1080/08982119908919259.

3. Martin P. The Market Opportunity. In: Building A Viable Regenerative Medicine

4. Hourd P, Chandra A, Medcalf N, Williams DJ. Regulatory challenges for the

5. Thurman-Newell JA, Petzing JN, Williams DJ. Quantification of biological variation in blood-based therapy - a summary of a meta-analysis to inform manufacturing in the

6. Domen J, Gandy K, Dalal J. Emerging uses for pediatric hematopoietic stem cells.

7. Auerbach $A D$, Landefeld CS, Shojania KG. The tension between needing to improve

8. Hopman RK, DiPersio JF. Advances in stem cell mobilization. Blood Rev. 2014;28(1):31-40. doi:10.1016/j.blre.2014.01.001.

9. Hung $\mathrm{Y}-\mathrm{C}$, Bauer J, Horsley $\mathrm{P}$, Waterhouse $\mathrm{M}$, Bashford J, Isenring E. Changes in

11. Marshall $M$, Pronovost $P$, Dixon-Woods $M$. Promotion of improvement as a science. cancer patients undergoing autologous peripheral blood stem cell transplantation. Support Care Cancer. 2013;21(6):1579-86. doi:10.1007/s00520-012-1698-y.

10. Winter JM, Jacobson P, Bullough B, Christensen AP, Boyer M, Reems J-A. Long-term effects of cryopreservation on clinically prepared hematopoietic progenitor cell products. Cytotherapy. 2014;16(7):965-75. doi:10.1016/j.jcyt.2014.02.005. 

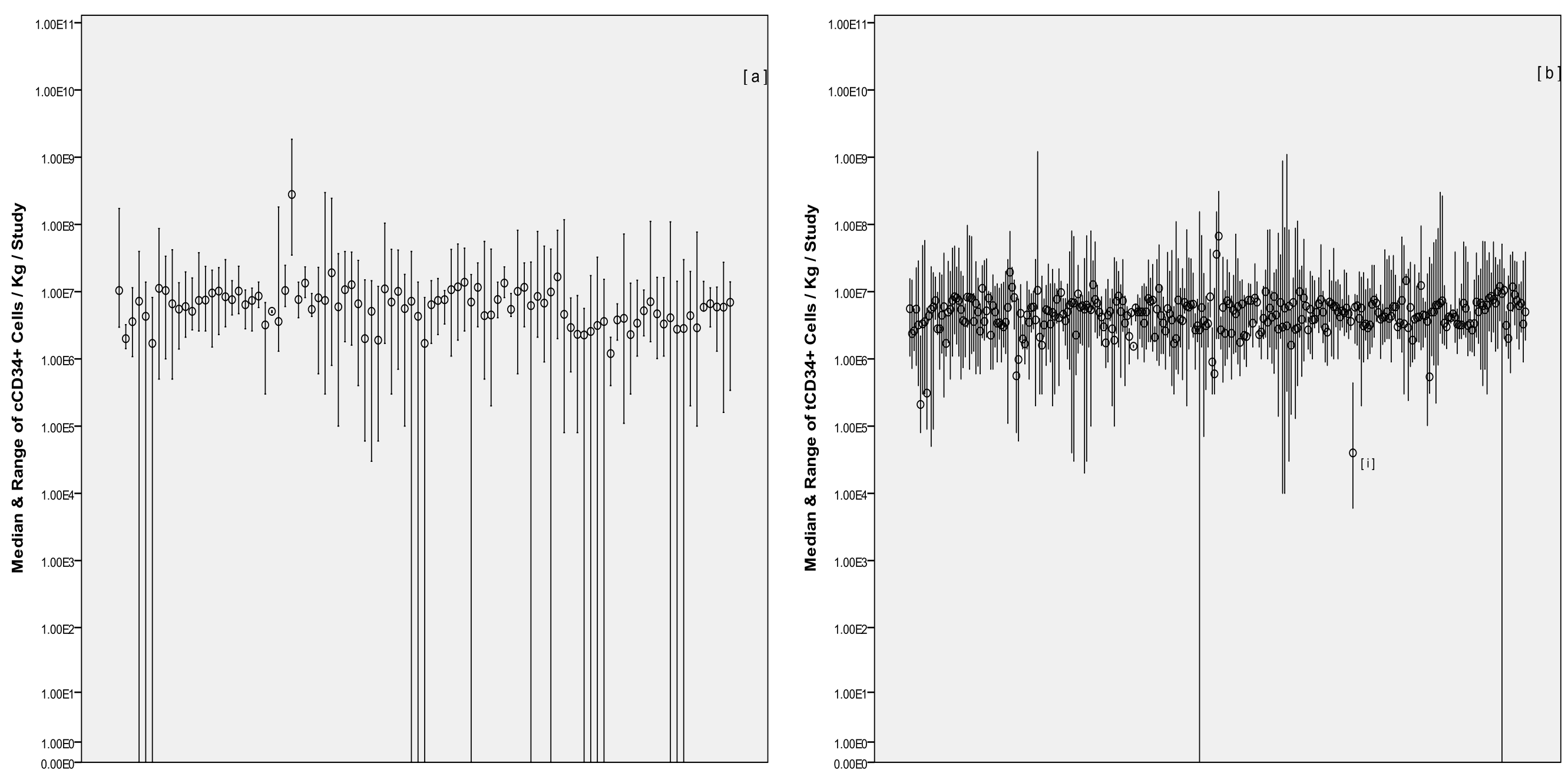
Peripheral

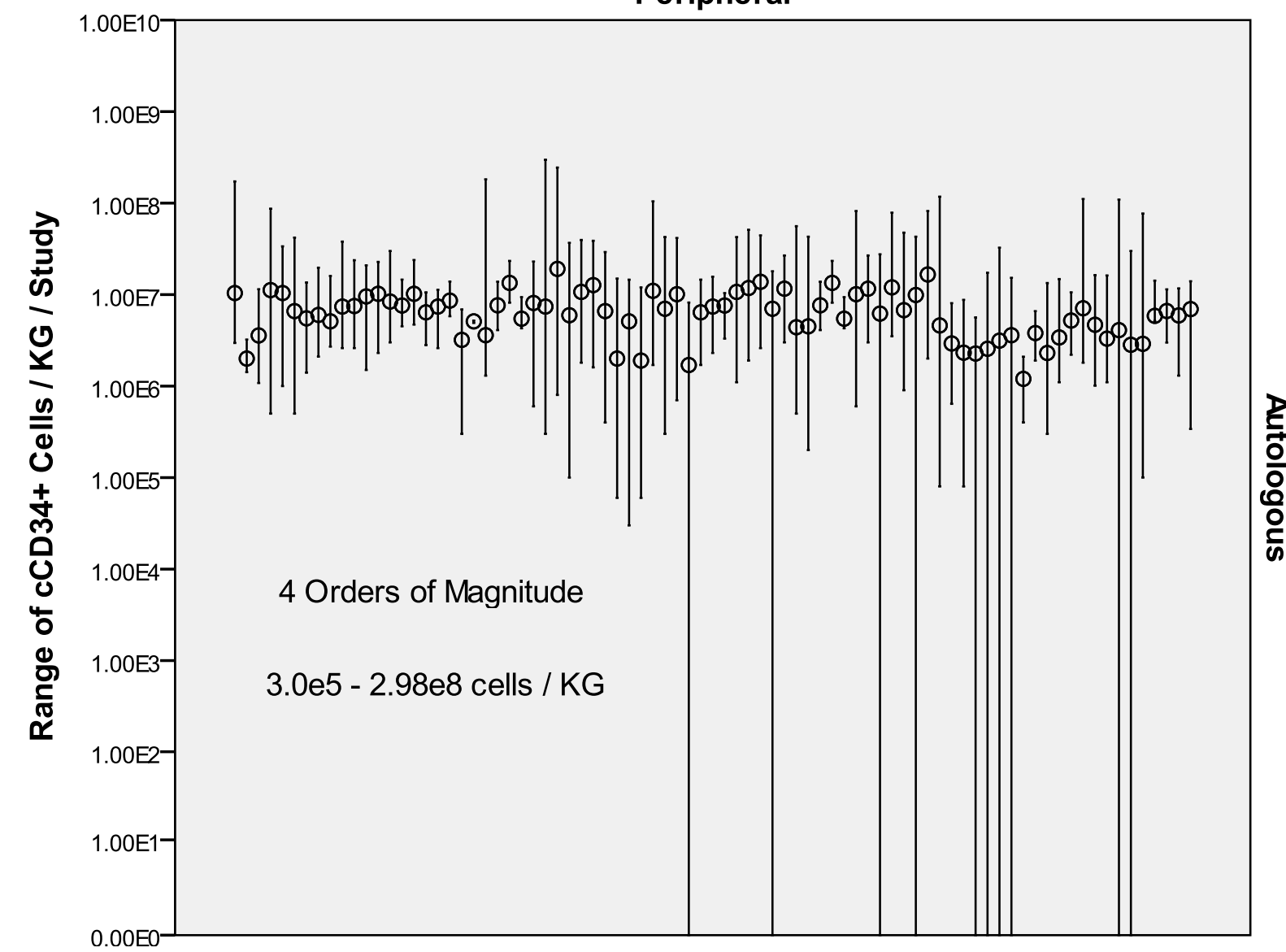


Peripheral

Bone Marrow

Mixed

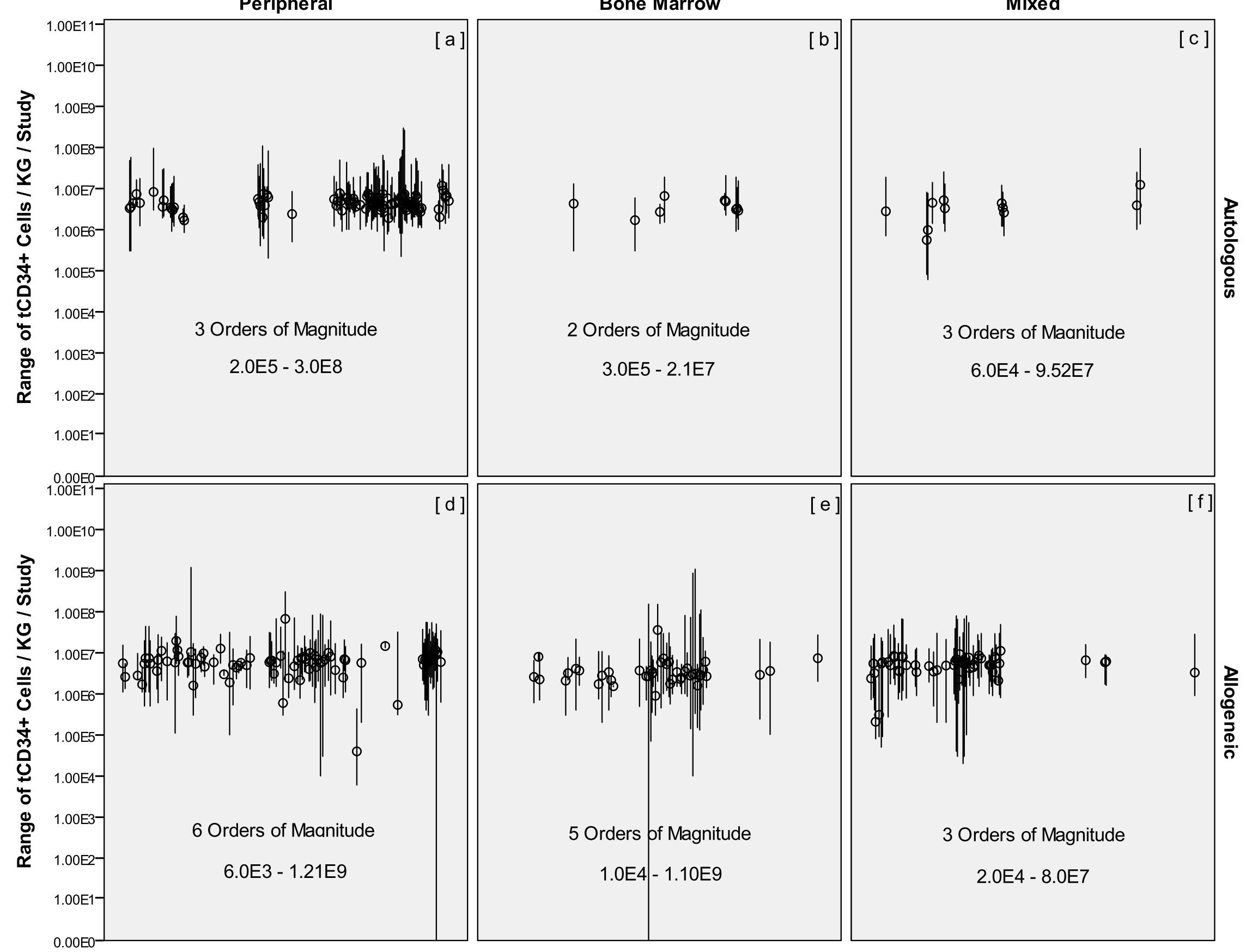



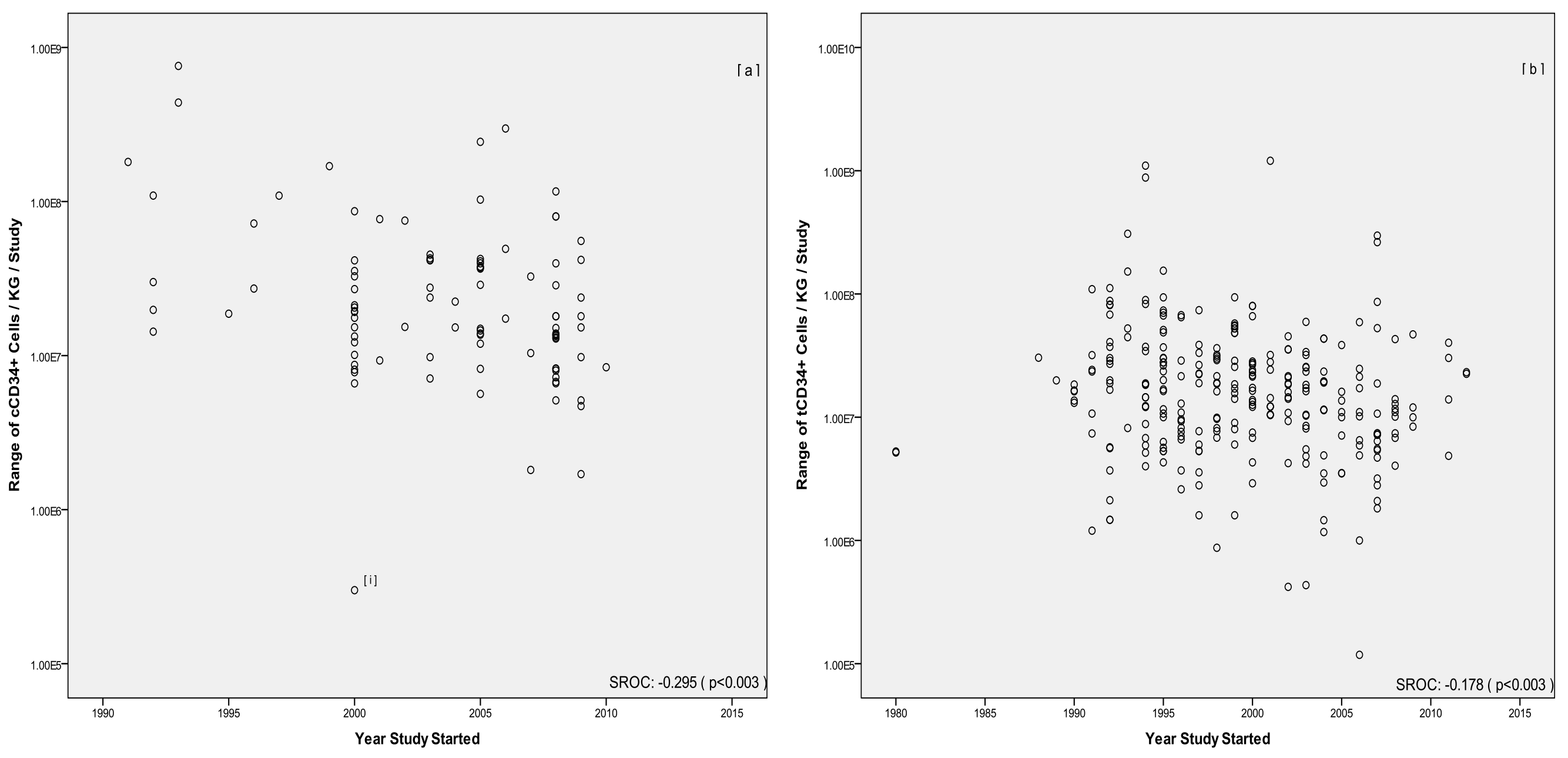


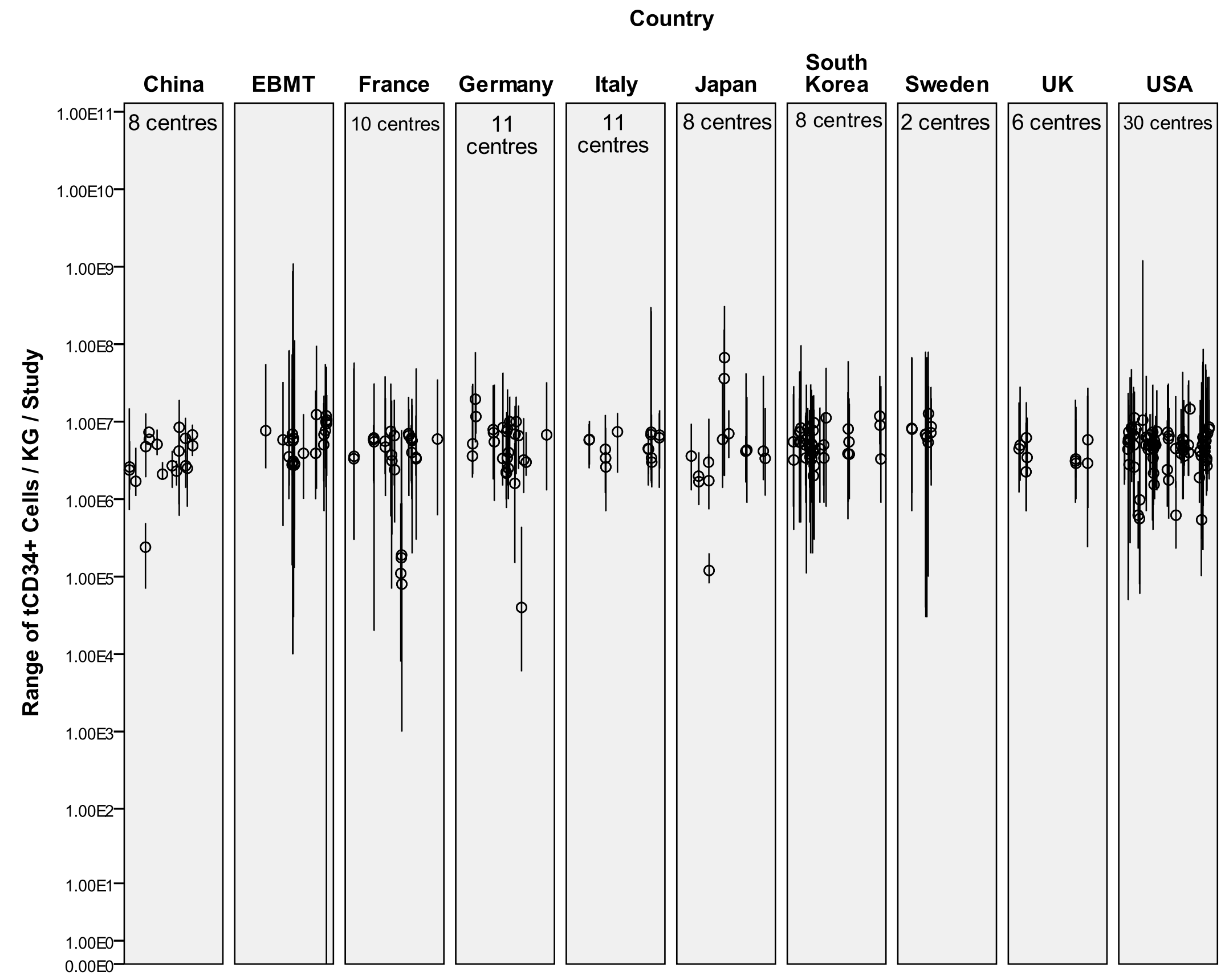




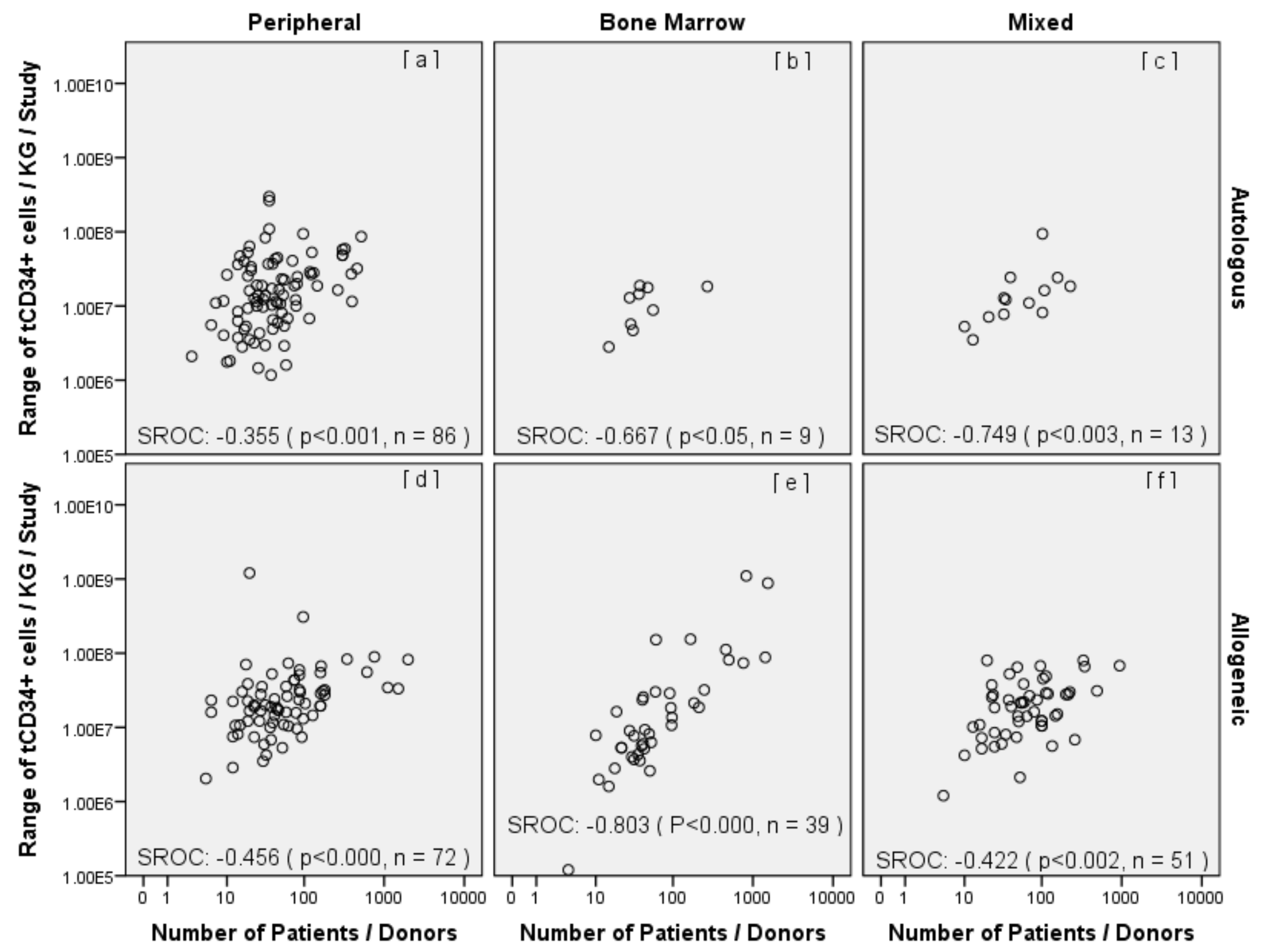




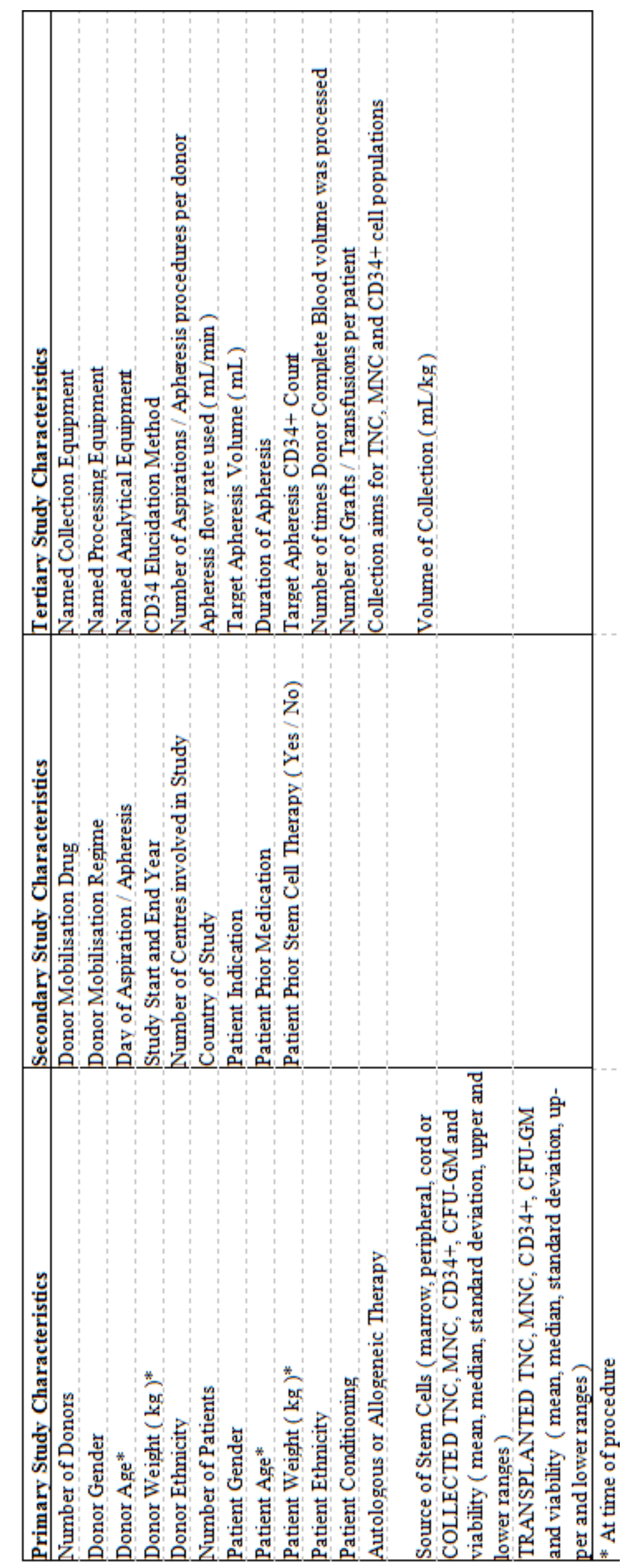

